\title{
Erratum to: The ventrolateral preoptic nucleus is required for propofol-induced inhibition of locus coeruleus neuronal activity
}

\author{
Yu Zhang ${ }^{1,2} \cdot$ Tian Yu ${ }^{1} \cdot$ Jie Yuan $^{1} \cdot$ Bu-Wei Yu ${ }^{2}$
}

Published online: 26 March 2016

(C) Springer-Verlag Italia 2016

Erratum to: Neurol Sci (2015) 36:2177-2184

DOI 10.1007/s10072-015-2292-0

Erroneously, the dosage level of propofol has been published with typo errors in the original publication, and inaccurate digital outputs existed in the figures. The dosage level of propofol should be read as $80 \mathrm{mg} / \mathrm{kg}$ instead of 20 $\mathrm{mg} / \mathrm{kg}$ in all occurrences. The correct figures are given below. The cable connection procedure before formal data recording was performed with rat under light anesthesia state and secured in a stereotaxic frame.

The online version of the original article can be found under doi:10.1007/s10072-015-2292-0.

$\mathrm{Bu}-\mathrm{Wei} \mathrm{Yu}$

ruijinyubuwei@163.com

Yu Zhang

gytzhangyu@163.com

1 Guizhou Key Laboratory of Anesthesia and Organ Protection, Affiliated Hospital of Zunyi Medical College, Zunyi,

Guizhou, People's Republic of China

2 Department of Anesthesiology, Ruijin Hospital, Shanghai JiaoTong University School of Medicine, Ruijin Er Road 197, Shanghai 200025, People's Republic of China 


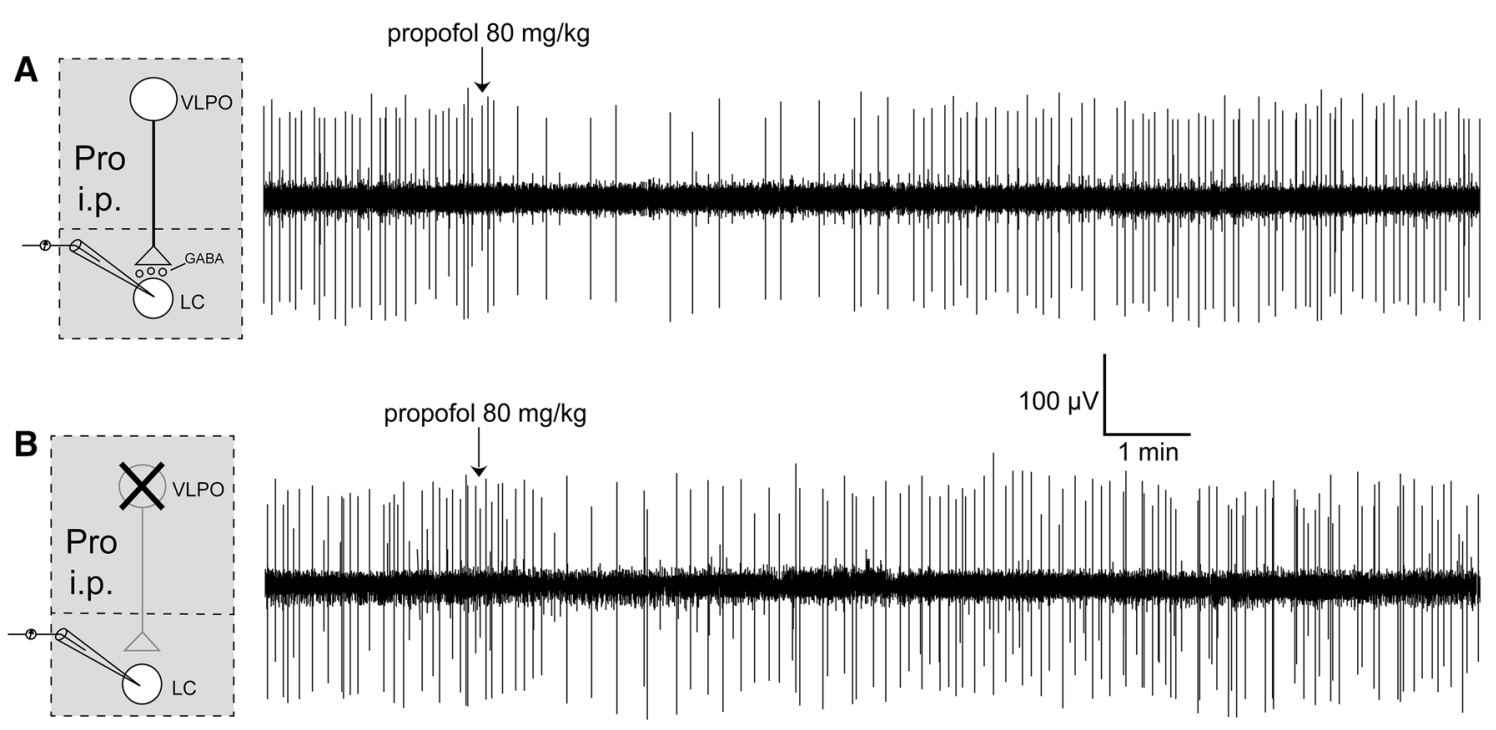

Fig. 4 Sample traces of spontaneous firings from LC neurons. a In normal rats, administration of propofol $(80 \mathrm{mg} / \mathrm{kg}$, IP) significantly decreased spontaneous firings of a LC neuron. b VLPO lesions reduced the inhibitory action of propofol on spontaneous firings of a LC neuron
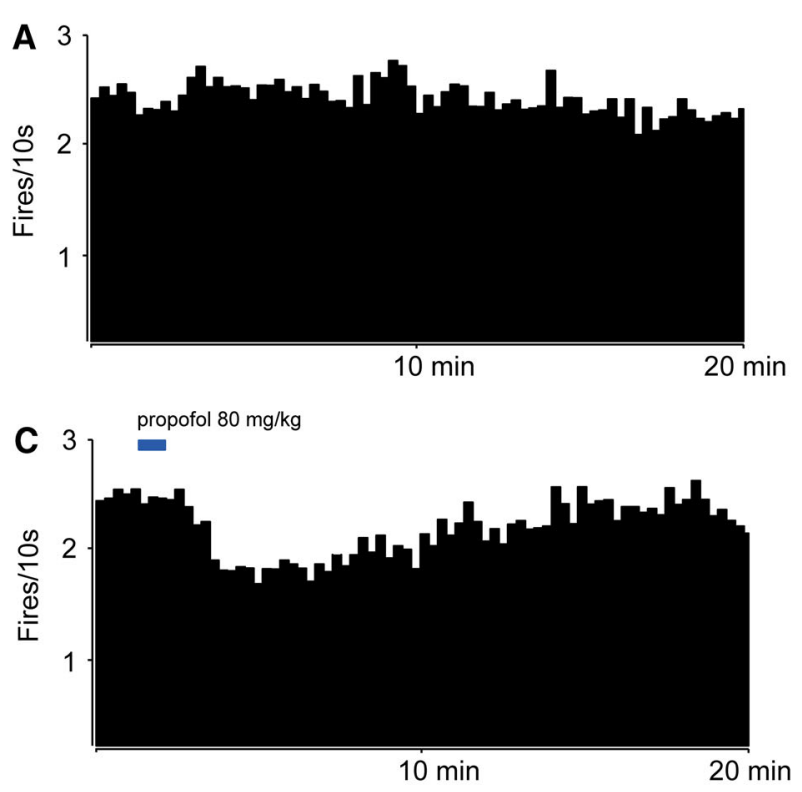

Fig. 5 Representative rate histograms (10 s bins) of spontaneous firing activity of LC neurons recorded in $20 \mathrm{~min}$ after propofol injection. a Rate histograms of spontaneous firing activity of LC neurons recorded in a normal awake rat. b Propofol $80 \mathrm{mg} / \mathrm{kg}$ significantly attenuated LC spontaneous activity of a normal awake rat. c VLPO lesion reduced the inhibitory effect of propofol on LC
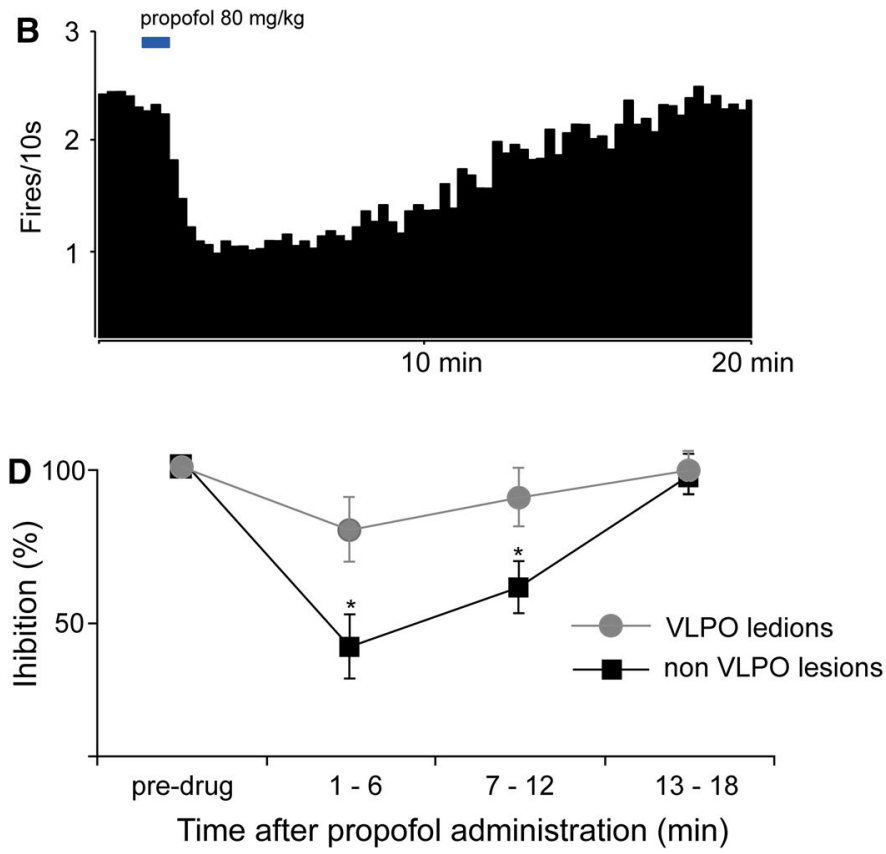

spontaneous activity and caused a much slower onset of this effect. d Inhibition ratio of propofol on spontaneous LC firing activity was recorded in both non-VLPO lesion rats and VLPO lesion rats. Values are mean \pm SD. $* P<0.05$ vs. non-VLPO lesion rats. Non-VLPO lesions, $n=7$; VLPO lesions, $n=5$ 

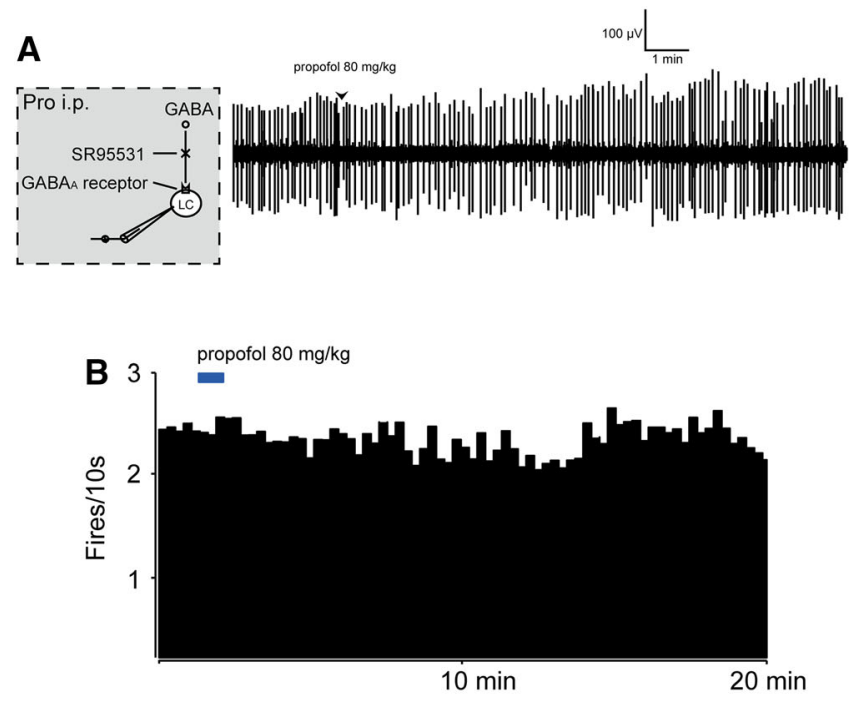

Fig. 6 Effects of propofol on spontaneous firing of LC neurons after microinjection of SR95531 into the LC $\mathbf{a}$ and $\mathbf{b}$ Administration of propofol $(80 \mathrm{mg} / \mathrm{kg}$, IP) did not observably affect the activities of the LC neurons.c There is no significant change of the latency to LORR

\section{C}

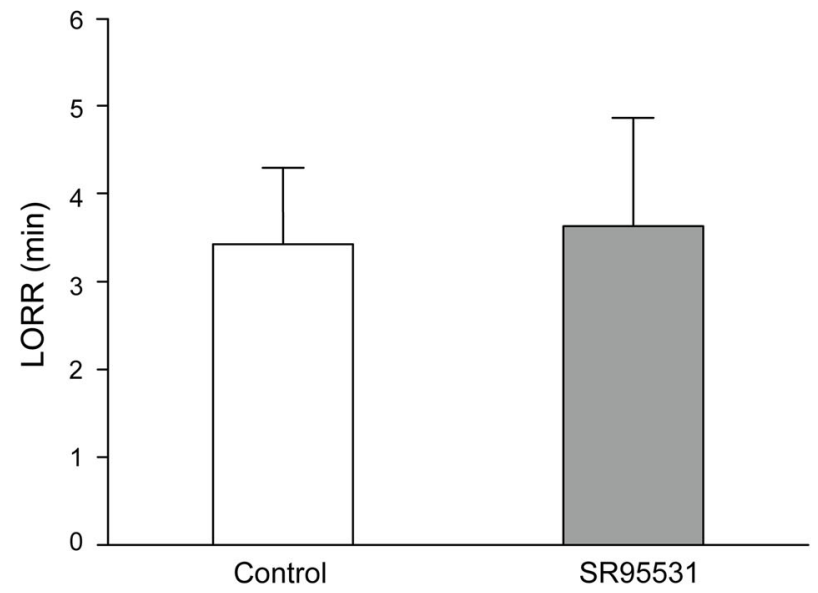

induced by propofol in rats with or without microinjection of SR95531 into the LC. Values are mean \pm SD. Control, $n=5$; SR95531, $n=5$ 